\title{
Plant movements as concept generators for deployable systems in architecture
}

\author{
S. Poppinga ${ }^{1,3,4}$ T. Masselter ${ }^{1,3,4}$, J. Lienhard ${ }^{2,3,4}$, S. Schleicher ${ }^{2,3,4}$, \\ J. Knippers ${ }^{2,3,4}$ \& T. Speck ${ }^{1,3,4}$ \\ ${ }^{I}$ Plant Biomechanics Group Freiburg, Botanic Garden, \\ Faculty of Biology, University of Freiburg, Germany \\ ${ }^{2}$ Institute of Building Structures and Structural Design (ITKE), \\ University of Stuttgart, Germany \\ ${ }^{3}$ Competence Network Biomimetics, Baden-Württemberg, Germany \\ ${ }^{4}$ Bionics Competence Network (BIOKON), Germany
}

\begin{abstract}
Plants, apparently not capable of complex movements, have always fascinated scientists when proving the contrary. A multitude of movements in plants have been revealed, showing a broad spectrum of motion sequences and underlying principles. Interestingly, many of these movements show high elasticity and flexibility of the respective structures and allow reversible deformations. With the investigation of suitable biological role models and the use of new construction materials, such as fibre-reinforced polymers (FRPs), the authors are developing deployable technical structures without local hinges. In this presentation the first steps of the applied biomimetic working process are described: the selection of role models, investigation and basic abstraction of plant movements. An overall screening through the plant kingdom has led to a wide-ranged matrix comprising many different types of plant movements, which constitutes the basis for our investigations. We distinguish between autonomous and non-autonomous movements. Active autonomous movements are characterized by motor organs, e.g. pulvini driven by a change of turgor pressure. Passive autonomous movements occur due to changing physical circumstances, e.g. bending through desiccation. Non-autonomous movements are mostly reversible deformations caused by a release of stored elastic energy after an external trigger or by direct application of mechanical forces. In a case study we applied morphological and anatomical investigations on the valvular pollination
\end{abstract}


mechanism of the Bird-Of-Paradise flower. A physical model as a first level abstraction step of the system is presented. In close collaboration between biologists and construction engineers this kinetic system is verified with the help of computer simulations and additional abstraction steps, which finally led to the construction of a bio-inspired demonstrator for technical applications.

Keywords: plant movements, biomimetics, deployable structures, pliable structures, elastic deformation, architecture.

\section{Introduction}

Deployability in architectural systems (e.g. large span umbrellas, blinds or even bascule bridges) is typically realized by technical hinges that often cause durability problems due to galling. Expensive maintenance works or even replacements of the respective parts are periodically necessary. Interestingly, deployability in plants (e.g. leaf orientation, blooming) functions with a complete absence of technical hinge analogies. Therefore, plant movements increasingly have become interesting as possible role models for developing biomimetic technical devices [1-3]. In the case study presented here the authors followed the methodology approach called the "Bottom-Up-Approach" (= biology push) as defined by the Plant Biomechanics Group Freiburg (Speck and Speck [4]), fig. 1.

A comprehensive compilation of plant movements serves as a basis for ongoing investigations of functional morphology and biomechanics, followed by several abstraction steps and finally a transfer into technical structures. Among the possible model systems different choice criteria are used. The examples chosen should potentially result in efficient solutions as to costs, energy and material solutions. Important for this is a good scalability of the concept generator, e.g. large bending radii in comparison to the thickness of the structure

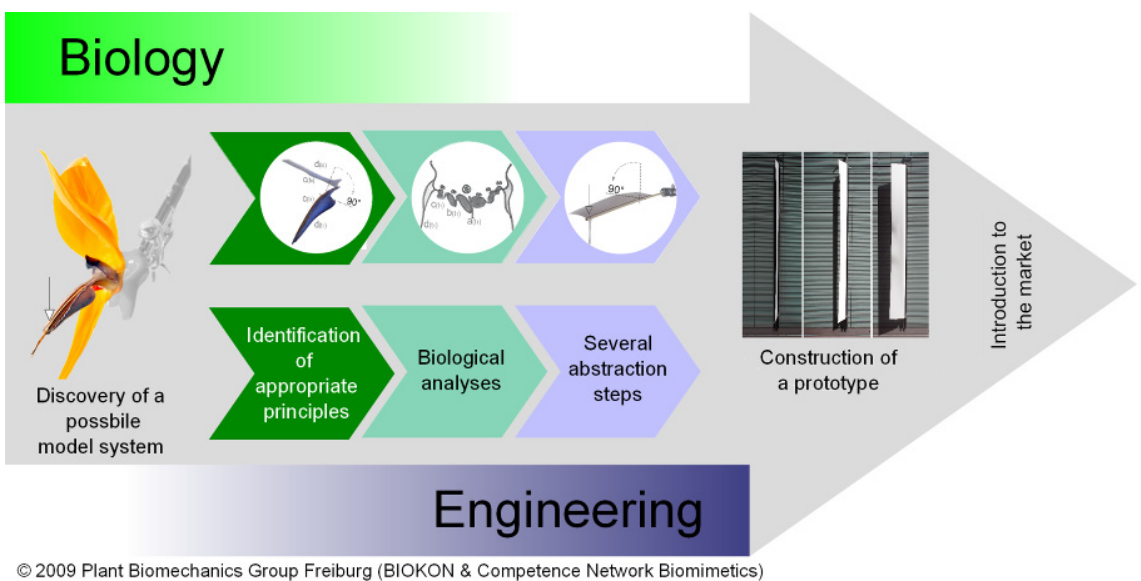

Figure 1: Bottom-Up-Approach for the development of a bio-inspired hingefree, deployable technical system. 
(to avoid high stresses); low actuation energy and avoidance of hinges are essential properties.

We found that many plant kinetic systems rely on material elasticity and flexibility, and these properties can be attained by new construction materials (like FRPs) according to various requirements (Matini [2]). This high potential has not yet found its use in construction and this has led us to expedite in this field we call elastic architecture.

\section{Model plant matrix}

The focus of current research mainly is on actuation systems found in so-called active or passive nastic plant movements that occur after reception of environmental stimuli (e.g. touch, change of humidity). Independent to the stimuli's direction, the resulting organ reactions follow a predetermined sequence due to their anatomy/morphology. Cell growth, a change of turgor pressure inside the cells (fig. 2a, b), cohesion forces and cell wall swelling or shrinking are the internal actuators of the movements (Burgert and Fratzl [3]).

These types of motions therefore are not generated by external factors and run autonomously. Interestingly, some types of plant movements do not run autonomously in contrast. They are based on reversible deformations which are actuated by external factors, such as an application of mechanical forces (fig. 2c, d), e.g. by visiting animals.

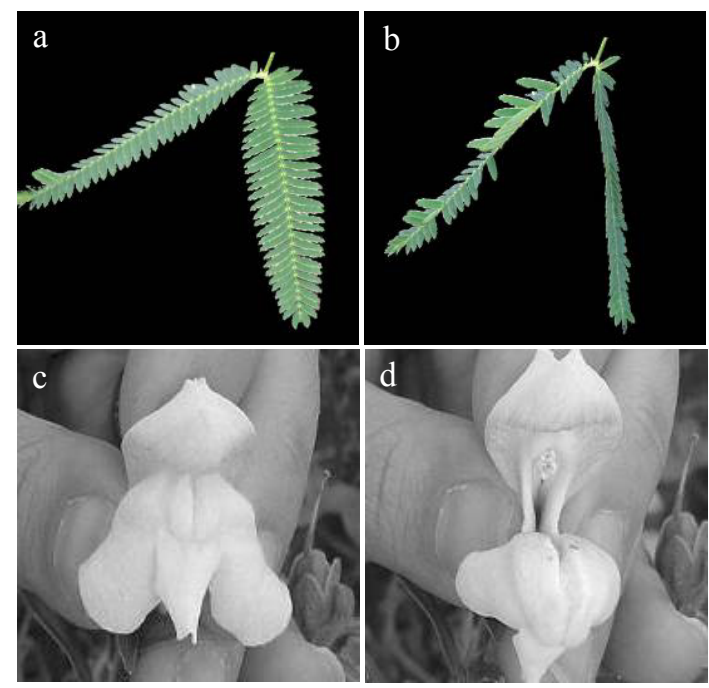

Figure 2: Autonomous, turgor-dependent leaflet movement in Mimosa spec. (a-b), non-autonomous, reversible elastic deformation in an Antirrhinum spec. flower due to the application of mechanical pressure (c-d, images taken from Matini [2]). 


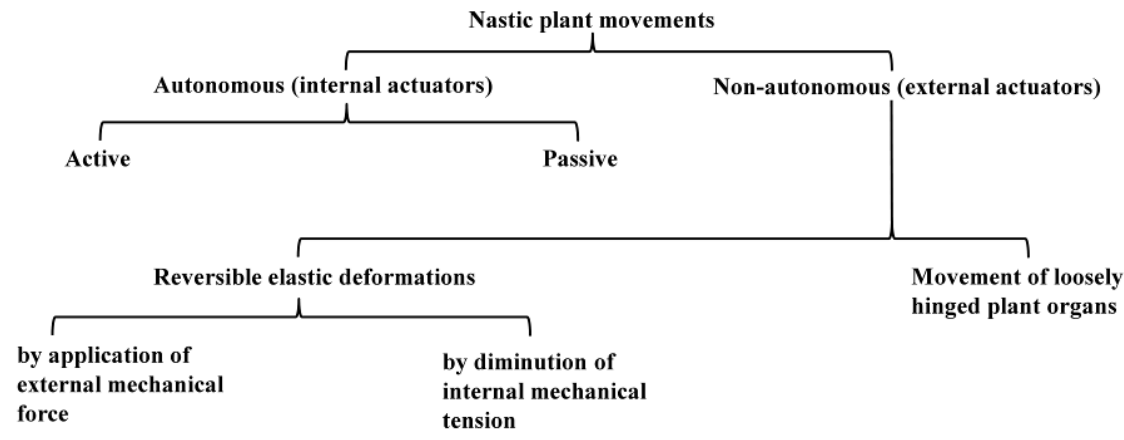

Figure 3: Proposal for an improved classification of nastic plant movement types.

As a basis for our biomimetic approach a model plant matrix was built up which allows us to present an improved classification of nastic plant movements (fig. 3) with non-autonomous systems included. They can be subdivided into reversible elastic deformations and movements of hinged organs. In both cases, external actuators like wind or visiting pollinators are needed to execute the movements. Reversible elastic deformations occur due to application of external mechanical force or due to diminution of internal mechanical tension. Both systems are presented in many pollination and seed dispersal mechanisms. Plant organs that are loosely (passively) hinged can be found in e.g. many orchid species, whose labellae function as pollinator attractants and move by slight wind force.

This screening categorises plant movements according to their principles of actuation, which allows us to use it as an inspiration basis for Bottom-UpApproaches. The following important basic questions about the possible concept generators can be answered easily:

1) What is moving? The organs included must be accurately defined (e.g. leaf, petal, anther). This can be important for investigations on temporarily developed structures, as in seasonally flowering plants.

2) Why is it moving? Besides the fundamental biological reasons for the motions (e.g. physiological processes involved in active autonomous systems), the underlying principles of the actuators must be defined to estimate the technical transfer possibilities (e.g. a transfer of turgor-dependent movements into pneumatic systems).

3) How is it moving? Information about e.g. bending radii, shape changes due to deformation and estimated actuation energies are important for planning possible technical transfers.

A different matrix by our project partners is based on a contrariwise approach: here, for optimization of established elastic kinematics, a search for construction techniques in potential plant role models is carried out (Top-DownApproach), see paper by Lienhard et al. (this conference). 


\section{The Bird-Of-Paradise as a case study}

As a first example in our project the valvular pollination mechanism of the BirdOf-Paradise (Strelitzia reginae, Strelitziaceae) was chosen. The Strelitzia's flower features two adnate petals that form a perch for pollinating birds $[5,6]$. When a bird sits on this perch to forage on nectar, its weight causes the perch to bend down. In a simultaneous movement, the petal lamina exposes the anthers and the style that are previously hidden and kept safe (Rowan [6]), fig. 4. When the bird flies away, the open state resets to the closed state due to the elastic properties of the perch. In this system, by application of a vertical force a horizontal movement is laid out, and the stored elastic energy is used to reset the system. It clearly can be considered as a non-autonomous, reversible deformation movement due to application of external mechanical force. It shows a distinct functional-morphological background with clearly defined actuating elements and mechanics.

In a first step the basic kinetic system was exposed by cutting off nondecisive flower parts. It was shown that only the basic structure/framework of the perch is essential for the deformation movement. Cut-off flower parts were the outer petals, the upper inner petal (not fused to the perch), the five stamens and the style (fig. 5).

Sections through the sheath of the perch reveal an almost monosymmetric build-up (fig.6). The lower rib actually is composed of two ribs, loosely connected via adjoining epidermal cells in a zigzag-pattern (for more details see Kronestedt and Walles [7]). Two additional lateral ribs on each side are connected via thin petal laminae. The upper lateral rib is fused to a large and

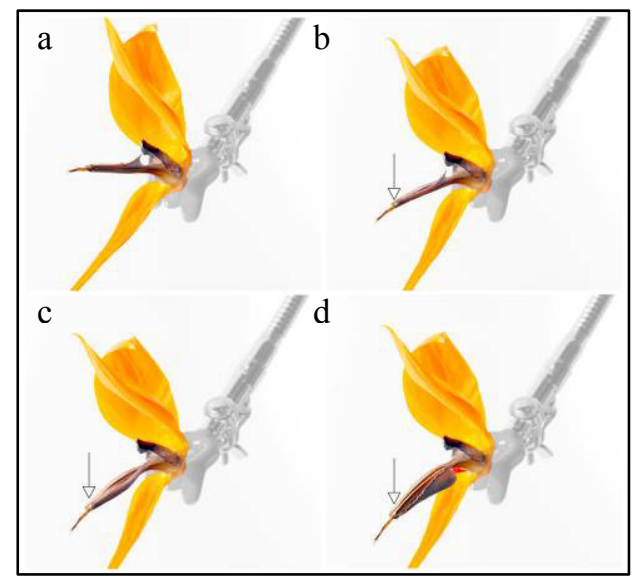

Figure 4: Motion sequence (a-d) of the kinetic system in the Strelitzia reginae flower. The arrow indicates the direction of the applied external mechanical force. 


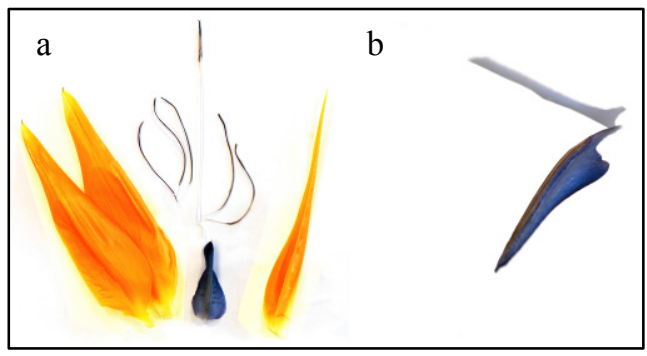

Figure 5: Cut-off, non decisive flower parts (a), basic kinetic system of the perch in closed and open positions (b).

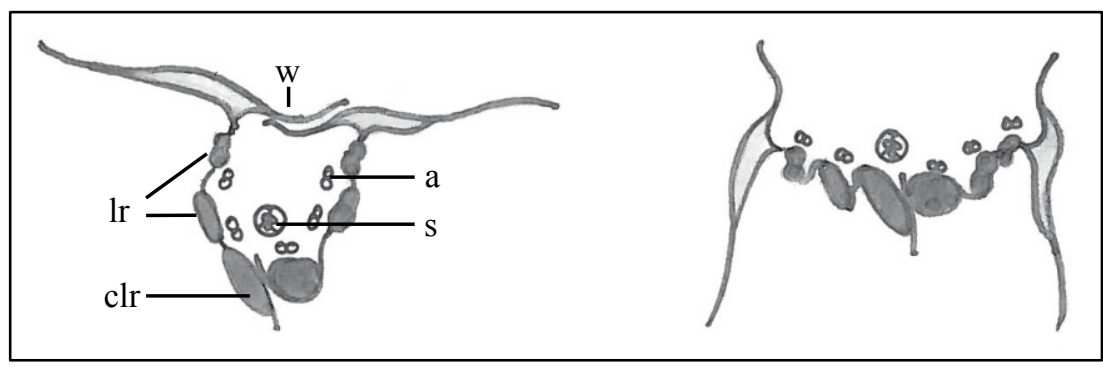

Figure 6: Schematic section through the closed perch (left) and in open position (right). clr: compound lower rib, lr: lateral rib, w: wing, a: anthers, s: style (here drawn with symbol for the ovary).

thickened lamina, with wing-like parts covering the anthers. Inside the cavity of the sheath, five anthers (consider the asymmetry here) and the style are loosely included. In the open position, all connected structures flap laterally in a specific manner.

\section{First abstraction step and outlook}

We found that a single upper lateral rib with the attached wing-lamina is also capable of the flapping movement. The perch as a whole seems to work as a composed system, in which the single rib-lamina flap movements together result in the valvular mechanism. This led us to rebuild this kinematic system in a very simple manner: we attached a paper strip horizontally on a flexible glass fibre rod. By bending the beam down, the paper strip flaps sideways and thus minimizes tension forces on the outer edge (Lienhard et al. [1]), fig. 7.

In further abstraction steps construction engineers and architects verify this finding with the help of orthographic projections and digital finite elements methods. The results were used for the construction of a first prototype of a hinge-less, stage-free flap. 


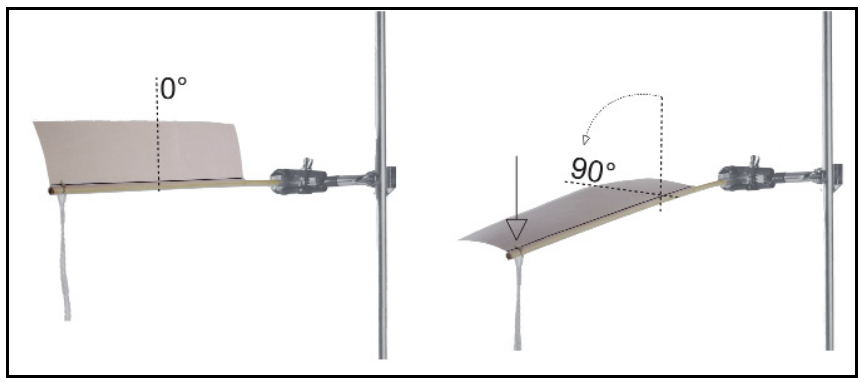

Figure 7: First level abstraction of the kinematic system in the Strelitzia flower.

\section{Acknowledgements}

The joint research project 'Deployable structures in architecture - flexible surface structures on the basis of bionic principles' is supported within the funding directive BIONA by the German Federal Ministry of Education and Research. We would like to thank our project partners for many helpful discussions.

\section{References}

[1] Lienhard, J., Poppinga, S., Schleicher, S., Masselter, T., Speck, T., Knippers, J., Abstraction of plant movements for deployable structures in architecture. Proc. of the 6th Plant Biomechanics Conf., ed. B. Thibaut, Cayenne, French Guyana, pp. 389-297, 2009.

[2] Matini, M.R., Biegsame Konstruktionen in der Architektur auf der Basis bionischer Prinzipien. PhD-thesis, Institut für Tragkonstruktionen und Konstruktives Entwerfen (ITKE): University of Stuttgart, 2007.

[3] Burgert, I., Fratzl, P., Actuation systems in plants as prototypes for bioinspired devices. Philosophical Transactions of the Royal Society A 367, pp. 1541-1557, 2009.

[4] Speck, T., Speck, O., Process sequences in biomimetic research. Design and Nature IV, ed. C.A. Brebbia, WIT Press: Southampton, pp. 3-11, 2008.

[5] Endress, P. K., Diversity and evolutionary biology of tropical flowers, Cambridge University Press, Cambridge Tropical Biology Series, 1994.

[6] Rowan, M. K., Bird pollination of Strelitzia. Ostrich 45, p. 40, 1974.

[7] Kronestedt, E., Walles, B., Anatomy of the Strelitzia reginae flower (Strelitziaceae). Nordic Journal of Botany 6 (3), pp. 307-320, 1986. 